\title{
STUDYING NANOCOMPOSITE FILMS WITH MATRIX-FORMING CARBON BY KELVIN PROBE FORCE MICROSCOPY
}

\author{
A. P. Rubshtein*, Yu. V. Korkh, A. B. Vladimirov, A. B. Rinkevich, S. A. Plotnikov \\ M.N. Miheev Institute of Metal Physics of Ural Branch of Russian Academy of Sciences, 18 S. Kovalevskoy st., 620990 \\ Ekaterinburg, Russian Federation

\begin{abstract}
*Corresponding author. E-mail: rubshtein@imp.uran.ru; address for correspondence: 18, ul. S. Kovalevskoy, 620990,
\end{abstract} \\ Ekaterinburg, Russian Federation. Tel.: +7 (343) 37835 37; Fax: +7 (343) 3745244.
}

Nanocomposite TiC/a-C and diamond-like carbon a-C films obtained by the vacuum ionplasma method have been studied by Kelvin Probe force microscopy. Films of thickness $200 \mathrm{~nm}$ were deposited on silicon wafers. The structure and composition of nanocomposite TiC/a-C films were varied by deposition conditions. It is shown that the average surface potential $V_{S P}^{a v}$ depends on the pulse frequency of graphite target sputtering during film deposition. The amount of $\mathrm{sp}^{3}$ bonded carbon on the film surface increases with scale up frequency. The $V_{S P}^{a v}$ of TiC/a-C nanocomposite films depends on the phase composition ( $\mathrm{Ti}, \mathrm{TiC}, \mathrm{a}-\mathrm{C})$ and their ratio. The numerically calculated volume fractions of the phases in the TiC/a-C films correlate with $V_{S P}^{a v}$.

Keywords: nanocomposite films, diamond-like carbon, titanium carbide, Kelvin probe force microscopy, average surface potential.

DOI: $10.17804 / 2410-9908.2015 .6 .104-113$

\section{References}

1. Robertson J. Diamond-like amorphous carbon. Materials Science and Engineering: R: Reports, 2002, vol. 37, iss. 4-6, pp. 129-281. DOI: 10.1016/S0927-796X(02)00005-0.

2. Rubstein A.P., Makarova E.B., Trakhtenberg I.Sh., Kudryavtseva I.P., Bliznets D.G., Philippov Yu.I., Shlykov I.L. Osseointegration of porous titanium modified by diamond-like carbon and carbon nitride. Diamond and Related Materials, 2012, vol. 22, pp. 128-135. DOI: $10.1016 /$ j.diamond.2011.12.030.

3. Trakhtenberg I.Sh., Vladimirov A.B., Plotnikov S.A., Rubshtein A.P., Vykhodets V.B., Bakunin O.M. Effect of adhesion strength of DLC to steel on the coating erosion mechanism. Diamond and Related Materials, 2001, vol. 10, iss. 9-10, pp. 1824-1828. DOI: 10.1016/S09259635(01)00430-7.

4. Musil J. Hard and superhard nanocomposite coatings. Surface and Coatings Technology, 2000, vol. 125, iss. 1-3, pp. 322-330. DOI: 10.1016/S0257-8972(99)00586-1.

5. Veprek S., Veprek-Heijman Maritza G.J., Karvankova P., Prochazka J. Different approaches to superhard coatings and nanocomposites. Thin Solid Films, 2005, vol. 476, iss. 1, pp. 1-29. DOI: $10.1016 /$ j.tsf.2004.10.053.

6. Yang Q., Zhao L.R. Microstructure, mechanical and tribological properties of novel multicomponent nanolayered nitride coatings. Surface and Coatings Technology, 2005, vol. 200, iss. 5-6, pp. 1709-1713. DOI: 10.1016/j.surfcoat.2005.08.087.

7. Dai W., Ke P., Moon M.W., Lee K.R., Wang A. Investigation of the microstructure, mechanical properties and tribological behaviors of Ti-containing diamond-like carbon films fabricated by a hybrid ion beam method. Thin Solid Films, 2012, vol. 520, iss. 19, pp. 6057-6063. DOI: 10.1016/j.tsf.2012.04.016.

8. Guo J., Hu X.J., Lu Y.H., Shen Y.G. Microstructure Evolution of nc-TiN/a-(C, CNx) Nanocomposite Films with Different Amorphous Phase Amounts. Procedia Engineering, 2013, vol. 67, pp. 388-396. DOI: 10.1016/j.proeng.2013.12.038.

Rubshtein A. P. et al. / Studying nanocomposite films with matrix-forming carbon by Kelvin probe force microscopy. 
9. Gulbinski W., Mathur S., Shen H., Suszko T., Gilewicz A., Warcholinski B. Evaluation of phase, composition, microstructure and properties in TiC/a-C:H thin films deposited by magnetron sputtering. Applied Surface Science, 2005, vol. 239, iss. 3-4, pp. 302-310. DOI: 10.1016/j.apsusc.2004.05.278.

10. Foong Y.M., Koh A.T.T., Lim S.R., Hsieh J., Chua D.H.C. Materials properties of $\mathrm{ZnO}$ /diamond-like carbon (DLC) nanocomposite fabricated with different source of targets. Diamond and Related Materials, 2012, vol. 25, pp. 103-110. DOI: 10.1016/j.diamond.2012.02.018.

11. Zhang S., Bui X.L., Jiang J., Li X. Microstructure and tribological properties of magnetron sputtered nc-TiC/a-C nanocomposite. Surface and Coatings Technology, 2005, vol. 198, iss. 1-3, pp. 206-211. DOI: 10.1016/j.surfcoat.2004.10.041.

12. Melitz W., Shen J., Kummel A.C., Lee S. Kelvin probe force microscopy and its application. Surface Science Reports, 2011, vol. 66, iss. 1, pp. 1-27. DOI: 10.1016/j.surfrep.2010.10.001.

13. Nonnenmacher M., O’Boyle M.P., Wickramasinghe H.K. Kelvin probe force microscopy. Applied Physics Letters, 1991, vol. 58, iss. 25, pp. 2921-2923. DOI: 10.1063/1.105227.

14. Trakhtenberg I.Sh., Gavrilov N.V., Emlin D.R., Plotnikov S.A., Vladimirov A.B., Volkova E.G., Rubshtein A.P. Nanocomposite vacuum-arc TiC/a-C:H coatings prepared using an additional ionization of acetylene. The Physics of Metals and Metallography, 2014, vol. 115, iss. 7, pp. 723-729. DOI: 10.1134/S0031918X14070102.

15. Trakhtenberg I.Sh., Vladimirov A.B., Rubstein A.P., Kuzmina E.V., Uemura K., Gontar A.G., Dub S.N. The analysis of microhardness measurement approach for characterization of hard coatings. Diamond and Related Materials, 2003, vol. 12, iss. 10-11, pp. 1788-1792. DOI: 10.1016/S0925-9635(03)00288-7.

16. Trakhtenberg I.Sh., Rubshtein A.P., Vladimirov A.B., Yugov V.A., Plotnikov S.A., Volkova E.G. Formation of morphology of surface of diamond-like films condenced upon arc deposition of graphite in a vacuum. The Physics of Metals and Metallography, 2005, vol. 100, iss. 1, pp. 56-60.

17. Xie W.G., Chen J., Chen J., Deng S.Z., She J.C., Xu N.S. Effect of hydrogen treatment on the field emission of amorphous carbon film. Journal of Applied Physics, 2007, vol. 101, iss. 8, pp. 084315-084500. DOI: 10.1063/1.2724426.

18. Ilie A., Hart A., Flewitt J., Robertson J., Milne W.I. Effect of work function and surface microstructure on field emission of tetrahedral amorphous carbon. Journal of Applied Physics, 2000, vol. 88, iss. 10, pp. 6002-6010. DOI: 10.1063/1.1314874.

Rubshtein A. P. et al. / Studying nanocomposite films with matrix-forming carbon by Kelvin probe force microscopy. 
Подана в журнал: 29.09.2015 г.

УДК 621.793;539.231

DOI: $10.17804 / 2410-9908.2015 .6 .104-113$

\title{
ИССЛЕДОВАНИЕ НАНОКОМПОЗИТНЫХ ПЛЕНОК С МАТРИЦЕОБРАЗУЮЩИМ УГЛЕРОДОМ МЕТОДОМ ЗОНДА КЕЛЬВИНА
}

\author{
А. П. Рубштейн*, Ю. В. Корх, А. Б. Владимиров, А. Б. Ринкевич, С. А. Плотников \\ Федеральное государственное бюджетное учреждение науки Институт физики металлов имени \\ М.Н. Михеева Уральского отделения Российской академии наук, ул. С.Ковалевской, 18, 620990, \\ Екатеринбург, Российская Федераџия
}

\begin{abstract}
*Ответственный автор. Электронная почта: rubshtein@imp.uran.ru, адрес для переписки: ул. С. Ковалевской, 18 , 620990, Екатеринбург, Российская Федерация. Телефон: +7 (343) 378-35-37; Факс: +7 (343) 374-52-44.
\end{abstract}

Методом зонда Кельвина изучены нанокомпозитные ТiC/a-C пленки и алмазоподобные углеродные а-C, полученные вакуумным ионно-плазменным методом. Пленки толщиной 200 нм осаждались на кремниевые пластины. Структура и состав нанокомпозитных $\mathrm{TiC} / \mathrm{a}-\mathrm{C}$ пленок варьировались условиями осаждения. Показано, что средний потенциал поверхности $V_{\Pi п}^{c p}$ а-С пленок зависит от частоты импульсов распыления графитовой мишени. При повышенной частоте $V_{\Pi П}^{c p}$ уменьшается, т.е. количество $\mathrm{sp}^{3}$ связанного углерода на поверхности пленки возрастает. Установлена зависимость $V_{\Pi \Pi}^{c p} \mathrm{TiC} / \mathrm{a}-\mathrm{C}$ пленок от фазового состава $(\mathrm{Ti}, \mathrm{TiC}, \mathrm{a}-\mathrm{C})$ и их соотношения. Найдена корреляция между $V_{I I}^{c p}$ и расчетными объемными долями фаз в $\mathrm{TiC} / \mathrm{a}-\mathrm{C}$ пленках.

Ключевые слова: нанокомпозитные пленки, алмазоподобныий углерод, карбид титана, метод зонда Кельвина, средний потенщиал поверхности.

\section{1. Введение}

Алмазоподобные углеродные пленки применяются для повышения износостойкости режущего инструмента, снижения коэффициентов трения деталей машин и механизмов, в магнитных устройствах для хранения данных, микро-электромеханических устройствах, биологических имплантатах благодаря их уникальным свойствам, таких как твердость, низкий коэффициент трения, оптическая прозрачность, биосовместимость [1-2]. Однако, большие внутренние напряжения сжатия в алмазоподобных пленках, возникающие вследствие их образования по механизму внутренней имплантации, приводят к плохой адгезии пленок к материалу подложки, что ограничивает их применение [3].

В течение последних лет класс сверхтвердых пленок существенно расширился за счет синтеза многокомпонентных нанокомпозитных покрытий, в частности пленок на основе нитридов и карбидов переходных металлов [4-6]. Широко исследуется физическая природа образования их структуры, влияние матрицы, легирующих элементов и размеров кристаллитов на их физико-механические и трибологические свойства. Интерес представляют композитные пленки, матрицей в которых является сверхтвердый алмазоподобный углерод (а-С, ta-C, a-C:H), а включениями - металлы $(\mathrm{Cu}, \mathrm{Ni})$, карбиды или нитриды переходных металлов $(\mathrm{Ti}, \mathrm{Cr}(\mathrm{C}, \mathrm{N}))$. Карбиды и нитриды переходных металлов характеризуются высокой твердостью, исключительной термостойкостью и хорошей коррозионной стойкостью.

Нанокомпозитные пленки с матрицеобразующим углеродом получают ионноплазменными методами путем распыления металлической и графитовой мишени или распылением многокомпонентной мишени [7-10]. Структура, физико-механические и трибологические свойства таких пленок зависят от относительного содержания в них аморфной матри-

Rubshtein A. P. et al. / Studying nanocomposite films with matrix-forming carbon by Kelvin probe force microscopy. 
цы углерода и нанокристаллических включений. Это соотношение влияет как на размер зерна, так и состояние аморфной матрицы. При увеличении содержания титана в $\mathrm{TiC} / \mathrm{a}-\mathrm{C}$ пленках от 16 до 48 ат. \% размер включений растет от 5 до 16 нм [11]. При этом рост содержания титана сопровождается снижением доли $\mathrm{sp}^{3}$ связей в алмазоподобной матрице. Аналогичная тенденция уменьшения размера включений карбида титана с увеличением доли углеродной матрицы отмечена в работе [9]. Прочностные свойства пленок в значительной степени обусловлены их структурой. Поэтому изучение структуры нанокомпозитных пленок, выявление основных факторов, влияющих на их формирование, является актуальной задачей в плане целенаправленного синтеза защитных материалов.

Для исследования структуры и состава композитных пленок широко используются традиционные методы: просвечивающая электронная микроскопия высокого разрешения [7-8], рентгеновская дифракция [9, 11], фотоэлектронная спектроскопия [7-8, 11], сканирующая зондовая микроскопия [9, 11], Рамановская спектроскопия [11]. Сканирующая зондовая микроскопия чаще всего используется в режиме атомно-силовой для определения параметров шероховатости поверхности пленок. Исследование же локальных электрических свойств поверхности с применением электросиловой зондовой микроскопии, в частности метода зонда Кельвина, в настоящее время в основном применяется для полупроводников, органических и биологических материалов [12]. Электросиловая зондовая микроскопия может дать дополнительную информацию о характеристиках композитных пленок, в состав которых входят фазы с различной электронной структурой. В настоящей работе методом зонда Кельвина, в основе которого лежит измерение контактной разности потенциалов между зондом и поверхностью пленки [13], проведено исследование нанокомпозитных TiC/a-C пленок, а также матрицеобразующих углеродных а-С структур, осажденных в различных условиях.

\section{2. Материалы и методы}

В качестве подложек для осаждения а-C и TiC/a-C пленок использовали кремниевые пластины толщиной 0,5 мм. Для очистки поверхности и достижения хорошей адгезии пленки к подложке пластины предварительно химически травили в $10 \%$-м растворе плавиковой кислоты в течение 5 мин. После химического травления пластины $\mathrm{Si}$ промывали в дистиллированной воде и спирте в ультразвуковой ванне. Перед помещением в рабочую камеру напылительной установки пластины сушили на воздухе при комнатной температуре.

Осаждение пленок проводили в установке УВНИИПА-1-001. Хороший контакт и теплоотвод во время конденсации пленок обеспечивались креплением подложек на водоохлаждаемом массивном медном держателе, установленном на механизме вращения. Перед осаждением пленок поверхность пластин подвергалась ионному травлению $(\mathrm{E}=4$ кэВ, $\mathrm{P}=4,2 \times 10^{-2}$ Па, $\mathrm{t}=30$ мин). Алмазоподобные пленки (а-C) осаждали распылением графитовой мишени при частоте импульсов дуги 5, 10, 15 и 25 Гц. Для получения нанокомпозитных пленок использовали титановый и графитовый катоды, которые распыляли одновременно. Титановый катод распыляли дуговым способом при постоянном токе дугового источника (55 A), а графитовый - импульсно-дуговым при тех же частотах. При используемых режимах и временах напыления толщина пленок составила 200 нм.

Состав пленок определяли по рентгеновским энергодисперсионным спектрам на сканирующем электронном микроскопе QUANTA 200 с приставкой EDAX. Анализировали пленки толщиной 1 мкм, осажденные на полированные медные подложки в тех же условиях, что и пленки на кремниевых пластинах. Состав определяли не менее чем на 5 участках пленки при варьировании размера области сканирования. Концентрация углерода и титана воспроизводилась в пределах $\pm 5 \%$.

Электрические свойства пленок изучали сканирующей зондовой микроскопией на атомно-силовом микроскопе Solver Next (Испытательный центр нанотехнологий и перспективных материалов ИФМ УрО РАН). Для этого использовали метод зонда Кельвина, в осно-

Rubshtein A. P. et al. / Studying nanocomposite films with matrix-forming carbon by Kelvin probe force microscopy. 
ве которого лежит измерение контактной разности потенциалов между проводящим наконечником (зондом) кантилевера и поверхностью пленки, зависящей от работы выхода электрона из материала наконечника и образца. Этот метод позволяет получать карту распределения потенциала по поверхности с высоким разрешением [12-13].

Исследование проводили на воздухе при комнатной температуре. Поверхность образца заземлялась медной пружиной. Использовали кантилевер HA_NC/Pt с зондом, покрытым пленкой из платины толщиной 20-30 нм. Высота зонда около $1 \overline{0}$ мкм, радиус кривизны головки не более 35 нм. Резонансная частота колебаний зонда 235 кГц. Карту распределения потенциала по поверхности получали в двухпроходном полуконтактном режиме для исключения влияния рельефа поверхности пленки на принимаемый сигнал. На первом проходе сканируемой строки определяли рельеф поверхности пленки по полуконтактному методу, во время второго прохода получали карту распределения потенциала по поверхности. При получении карты распределения потенциала по поверхности расстояние между сканируемой поверхностью и зондовым датчиком поддерживалось постоянным (50 нм). Колебания кантилевера во втором проходе возбуждались электрически путем приложения к зонду переменного напряжения $\mathrm{V}_{\mathrm{I}}= \pm 2 \mathrm{~B}$. Скорость сканирования $-0,5$ Гц, области сканирования (10x10) мкм и (1x1) мкм, шаг сканирования 20 и 2 нм соответственно.

Интегральные профили распределения разности потенциалов строили по прямоугольной области полученных изображений. Математическую обработку профилей проводили с помощью программного обеспечения микроскопа. Для характеристики пленок использовали среднее значение потенциала поверхности $V_{\text {ПП }}^{c p}$. Средние значения потенциала поверхности воспроизводились в пределах $\pm 11 \%$.

\section{3. Результаты и обсуждение}

Анализ состава пленок по рентгеновским энергодисперсионным спектрам показал нелинейную зависимость содержания титана в $\mathrm{TiC} / \mathrm{a}-\mathrm{C}$ пленках от частоты импульсов дуги при распылении графита (рис. 1, кривая 1). Ранее на основе диаграммы состояния системы углерод-титан были найдены соотношения объемов фаз в композите $\mathrm{TiC} / \mathrm{a}-\mathrm{C}$ в зависимости от весовой концентрации углерода и выведено уравнение для определения объемной доли карбида титана [14]. Соотношения получены в предположении, что при образовании композита в условиях, когда весовая доля углерода составляет 0,2 , он состоит из одной фазы карбида титана ТiC. При содержании углерода, недостаточном для образования стехиометрического карбида титана $(<0,2)$, пленочный композит состоит из частиц титана и его карбида. При содержании углерода более 0,2 происходит формирование аморфной матрицы с включениями TiC. Наличие частиц $\mathrm{Ti}, \mathrm{TiC}_{\mathrm{x}}$ и аморфного углерода было подтверждено ранее при исследовании пленок методом просвечивающей электронной микроскопии. Условия получения пленок, их состав и расчетная объемная доля карбида титана в пленках приведены в табл. 1. На рис. 1 (кривая 2) представлена зависимость объемной доли титана от частоты, стрелками показаны области образования ( $\mathrm{Ti}+\mathrm{TiC})$ и $(\mathrm{a}-\mathrm{C}+\mathrm{TiC})$.

Различное соотношение фаз в нанокомпозитной пленке, отличающихся электронной структурой, неизбежно оказывает влияние на ее электрические свойства. Присутствие матрицеобразующего углерода осложняет задачу изучения композитных пленок, так как свойства углеродной матрицы, имеющей алмазоподобную структуру, определяются соотношением sp ${ }^{2} / \mathrm{sp}^{3}$ связей между атомами углерода, которое зависит от условий осаждения [1], в частности от частоты импульсов при конденсации пленок [15]. Поэтому методом зонда Кельвина, в дополнение к нанокомпозитным пленкам, были изучены а-С пленки, полученные при импульсно дуговом распылении графита без распыления титановой мишени.

Rubshtein A. P. et al. / Studying nanocomposite films with matrix-forming carbon by Kelvin probe force microscopy. 
Таблица 1 - Условия осаждения ТiС/а-С пленок и их состав

\begin{tabular}{|c|c|c|}
\hline Частота $\mathbf{f}, \boldsymbol{\Gamma} \mathbf{u}$ & Весовая доля титана/углерода $\left(\mathbf{C}_{\mathbf{T}} / \mathbf{C}_{\mathbf{C}}\right)$ & Объемная доля карбида титана \\
\hline 5 & $0,89 / 0,11$ & 0,53 (остальное титан) \\
\hline 10 & $0,82 / 0,18$ & 0,92 \\
\hline 15 & $0,75 / 0,25$ & 0,91 (остальное a-C) \\
\hline 20 & $0,66 / 0,36$ & 0,74 (остальное a-C) \\
\hline 25 & $0,49 / 0,51$ & 0,49 (остальное a-C) \\
\hline
\end{tabular}

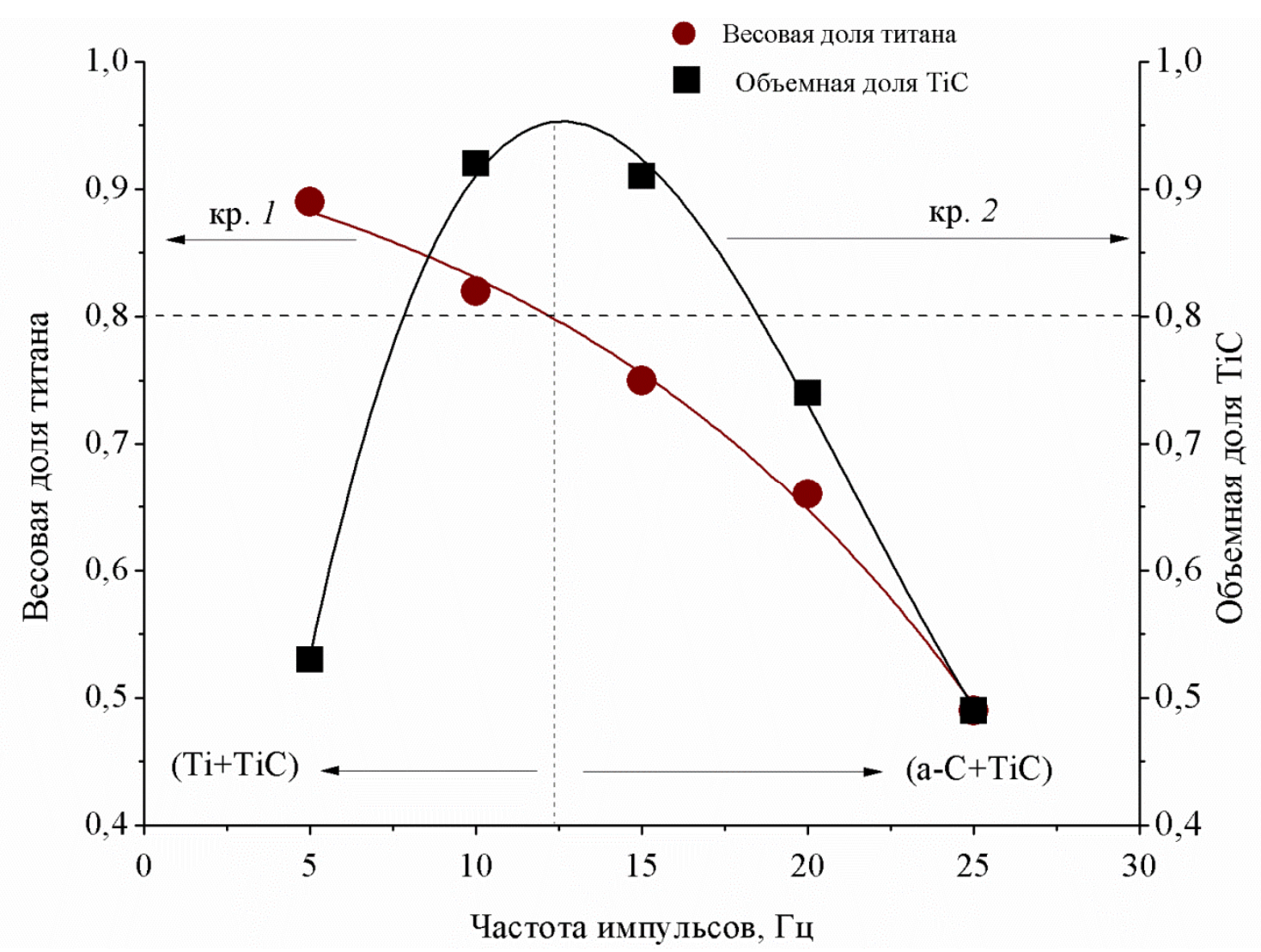

Рис. 1. Зависимости весовой доли титана (левая шкала, кривая 1) и объемной доли карбида титана ТіС (правая шкала, кривая 2) от частоты импульсов при распылении графитовой мишени. Стрелками обозначены области образования ( $\mathrm{Ti}+\mathrm{TiC})$ и $(\mathrm{a}-\mathrm{C}+\mathrm{TiC})$ пленок

Предварительно были получены изображения рельефа поверхности а-С пленок на атомно-силовом микроскопе при сканировании области размером (10х10) мкм. Наличие больших выступов на их поверхности (рис. $2 a$ ) связано с механизмом формирования алмазоподобного углерода и отсутствием магнитной сепарации $\mathrm{C}^{+}[16]$. Для исключения влияния выступов при двухпроходном сканировании выбирались однородные области размером (1x1) мкм (рис. 2 б). Типичная карта распределения потенциалов по поверхности алмазоподобной пленки представлена на рис. 2 в. Равномерный контраст свидетельствует об однородности пленки. Наличие областей с различными связями между атомами углерода $\left(\mathrm{sp}^{2}\right.$ и $\left.\mathrm{sp}^{3}\right)$, отличающихся шириной запрещенной зоны, не наблюдается, так как их размеры составляют несколько межатомных расстояний. Средние значения потенциала поверхности $\left(V_{\Pi \Pi}^{c p}\right)$ для аС пленок приведены в табл. 2. Наблюдается тенденция снижения $V_{\Pi \Pi}^{c p}$ с ростом частоты импульсов, что, вероятнее всего, связано с возрастанием количества $\mathrm{sp}^{3}$ связанного углерода [17]. Известно, что ширина запрещенной зоны в а-C пленках зависит от соотношения $\mathrm{sp}^{2} / \mathrm{sp}^{3}$ связей между атомами углерода. Рост количества $\mathrm{sp}^{3}$ связанного углерода сопровождается

Rubshtein A. P. et al. / Studying nanocomposite films with matrix-forming carbon by Kelvin probe force microscopy. 
open-recess journal

уширением запрещенной зоны и, соответственно, увеличением работы выхода электрона $[1,18]$. Согласно фундаментальным принципам, на которых основан метод зонда Кельвина, между проводящим наконечником кантилевера и образцом измеряется контактная разность потенциалов, определяемая как $\mathrm{V}_{\text {Крп }}=\left(\varphi_{\text {нак }}-\varphi_{\text {обр }}\right) / \mathrm{e}$, где $\varphi_{\text {нак }}-$ работа выхода электрона для наконечника, $\varphi_{\text {обр }}$ - работа выхода электрона для образца, е - элементарный заряд. При

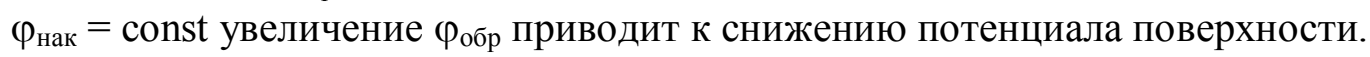

Аналогичная процедура выбора участка сканирования для двухпроходного режима проводилась для ТіС/a-C пленок, так как образование выступов наблюдалось и на их поверхности. Данные по измерению $V_{\text {Пп }}^{c p}$ для этих пленок приведены в табл. 2. Как видно из таблицы, композитные TiC/a-C пленки, в отличие от а-С, имеют зависимость $V_{\Pi \Pi}^{c p}(f)$, содержащую

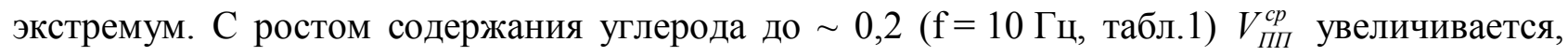
дальнейший рост доли углерода в нанокомпозитной пленке (увеличение $\mathrm{f}$, табл.1) приводит к падению $V_{\text {Пп }}^{c p}$ (рис. 3).

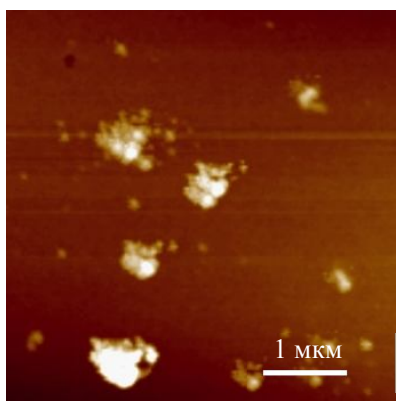

$a$

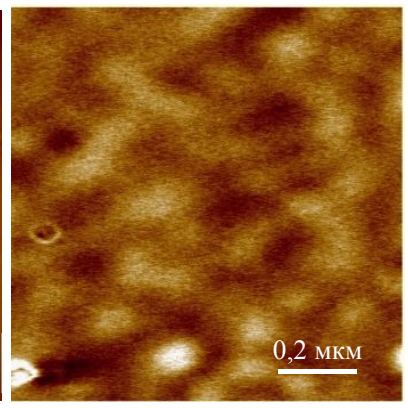

$\sigma$

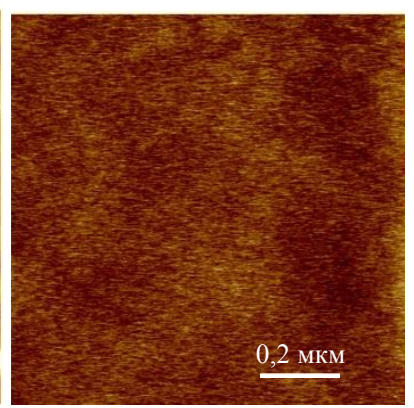

B

Рис. 2. АСМ изображение поверхности $(a, \sigma)$ и распределение потенциала по поверхности (в) а-С пленки ( $\mathrm{f}=15$ Гц)

Таблица 2 - Средний потенциал поверхности а-C и TiC/a-C пленок

\begin{tabular}{|c|c|c|}
\hline \multirow{2}{*}{ Частота f, Гц } & \multicolumn{2}{|c|}{$V_{\Pi \Pi}^{c p}(\mathrm{MB})$} \\
\cline { 2 - 3 } & $\mathbf{a}-\mathbf{C}$ & TiC/a-C \\
\hline 5 & 2,0 & 1,4 \\
\hline 10 & 2,0 & 3,3 \\
\hline 15 & 1,4 & 2,5 \\
\hline 25 & 1,4 & 1,6 \\
\hline
\end{tabular}

Изменение $V_{I I I}^{c p}$ TiC/a-C пленок связано как с изменением их фазового состава, так и размера зерна. При малой концентрации углерода пленки состоят из частиц титана и карбида титана. Повышение концентрации углерода путем увеличения f до 10 Гц приводит к преобладанию в пленке частиц ТіС и росту $V_{\Pi п}^{c p}$ до 3,3 мВ (табл. 2). Для этих пленок наблюдался наибольший контраст на карте распределения потенциала по поверхности (рис. 4 б).

При $\mathrm{f}>10$ Гц углерод в пленке становится матрицеобразующим, т.е. между частицами карбида титана находится аморфная матрица. Увеличение доли аморфного углерода в TiC/a-C приводит к падению $V_{\Pi П}^{c p}$ до 1,6 мB, что сравнимо по величине для а-С, осажденной при той же частоте (табл. 2). Значение $V_{\text {Пп }}^{c p}$, близкое по величине для а-С, получено для композитной пленки с довольно большой объемной долей карбида титана $-0,49$ (рис. 2 , кривая 2). Это, вероятнее всего, связано с уменьшением размера карбидных включений при

Rubshtein A. P. et al. / Studying nanocomposite films with matrix-forming carbon by Kelvin probe force microscopy. 
opent-access jurrnal

возрастании доли аморфной матрицы [11]. При малых размерах включений в пленке основной вклад в среднюю разность потенциалов дает сплошная углеродная матрица.

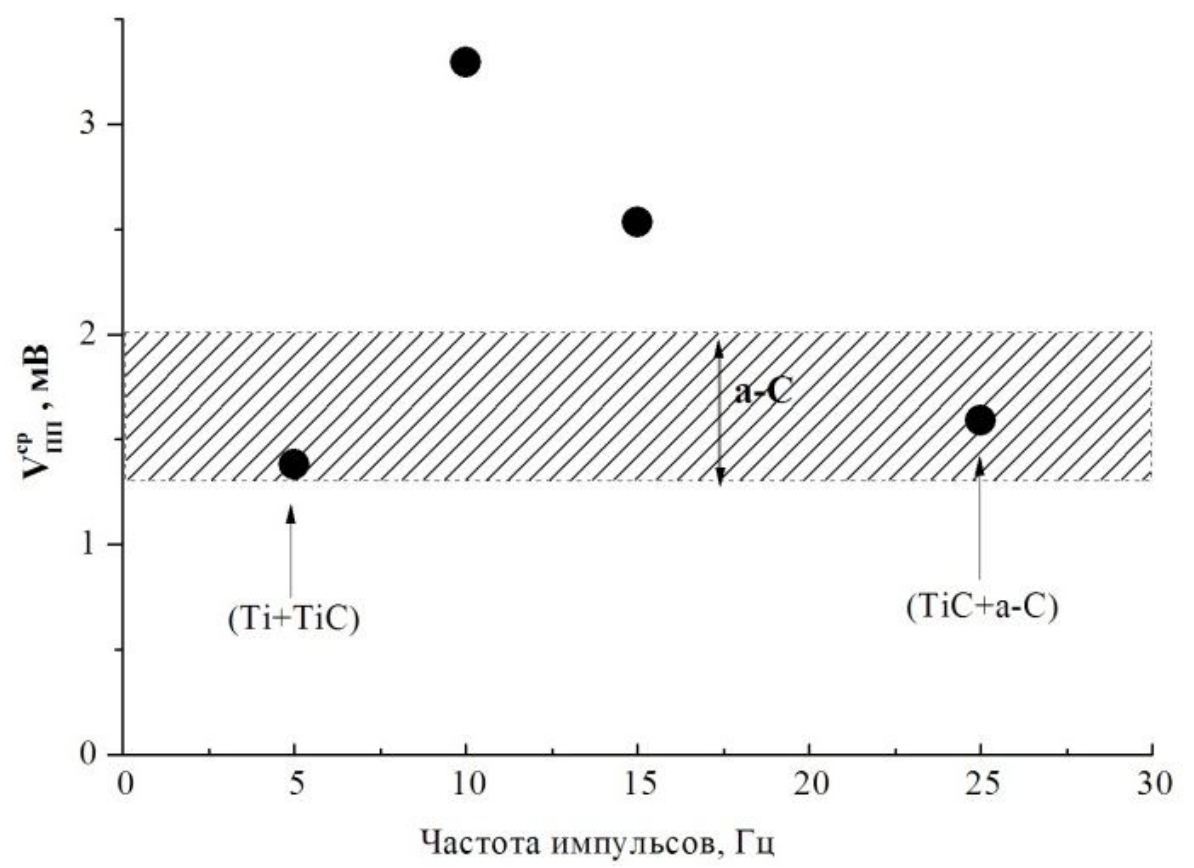

Рис. 3. Зависимость среднего потенциала поверхности от частоты импульсов для нанокомпозитных TiC/a-C пленок

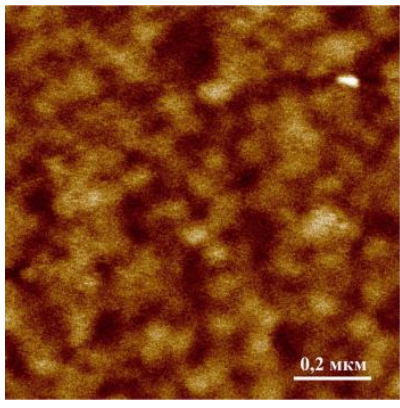

$a$

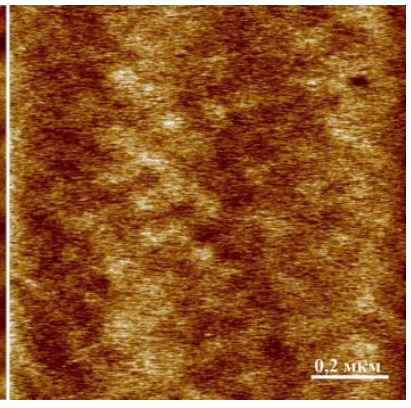

6

Рис. 4. АСМ изображение поверхности ( $a$ ) и распределение потенциала по поверхности (б) $\mathrm{TiC} / \mathrm{a}-\mathrm{C}$ пленки $(\mathrm{f}=10$ Гц $)$

\section{4. Заключение}

В работе апробирован метод зонда Кельвина для исследования нанокомпозитных TiC/a-C пленок и матрицеобразующих а-С структур, полученных в аналогичных условиях. Показано, что метод зонда Кельвина, применим для исследования структурных особенностей композитных пленок, в состав которых входит несколько фаз. Найдена корреляция между расчетной объемной долей фаз в ТiC/a-C пленке и средним потенциалом поверхности пленки.

\section{Благодарность}

Работа выполнена в рамках темы "Спин" № 1201463330 при частичной поддержке УрО РАН, проект №15-9-2-12".

Rubshtein A. P. et al. / Studying nanocomposite films with matrix-forming carbon by Kelvin probe force microscopy. 


\section{Литература}

1. Robertson J. Diamond-like amorphous carbon // Materials Science and Engineering R. 2002. - Vol. 37, iss. 4-6. - P. 129-281. - DOI: 10.1016/S0927-796X(02)00005-0.

2. Osseointegration of porous titanium modified by diamond-like carbon and carbon nitride / A. P. Rubstein, E. B. Makarova, I. Sh. Trakhtenberg, I. P. Kudryavtseva, D. G. Bliznets, Yu. I. Philippov, I. L. Shlykov // Diamond and Related Materials. - 2012. - Vol. 22 - - P. 128-135. - DOI: 10.1016/j.diamond.2011.12.030.

3. Effect of adhesion strength of DLC to steel on the coating erosion mechanism / I. Sh. Trakhtenberg, A. B. Vladimirov, S. A. Plotnikov, A. P. Rubshtein, V. B. Vykhodets, O. M. Bakunin // Diamond and Related Materials. - 2001. - Vol. 10, iss. 9-10. - P. 1824-1828. DOI: $10.1016 / \mathrm{S} 0925-9635(01) 00430-7$.

4. Musil J. Hard and nanocomposite coatings // Surface and Coatings Technology. - 2000. Vol. 125, iss. 1-3. - P. 322-330. - DOI: 10.1016/S0257-8972(99)00586-1.

5. Different approaches to superhard coatings and nanocomposites / S. Veprek, M. G. J. Veprek-Heijman, P. Karvankova, J. Prochazka // Thin Solid Films. - 2005. - Vol. 476, iss. 1. - P. 1-29. - DOI: 10.1016/j.tsf.2004.10.053.

6. Yang Q., Zhao L. R. Microstructure, mechanical and tribological properties of novel multicomponent nanolayered nitride coatings // Surface and Coatings Technology. - 2005. - Vol. 200, iss. 5-6. - P. 1709-1713. - DOI: 10.1016/j.surfcoat.2005.08.087.

7. Investigation of the microstructure, mechanical properties and tribological behaviors of Ticontaining diamond-like carbon films fabricated by a hybrid ion beam method / W. Dai, P. Ke, M. W. Moon, K. R. Lee, A. Wang // Thin Solid Films. - 2012. - Vol. 520, iss. 19. - P. 6057-6063. - DOI: 10.1016/j.tsf.2012.04.016.

8. Microstructure Evolution of nc-TiN/a-(C, CNx) Nanocomposite Films with Different Amorphous Phase Amounts / J. Guo, X. J. Hu, Y. H. Lu, Y. G. Shen // Procedia Engineering. 2013. - Vol. 67. - P. 388-396. - DOI: 10.1016/j.proeng.2013.12.038.

9. Evaluation of phase, composition, microstructure and properties in TiC/a-C:H thin films deposited by magnetron sputtering / W. Gulbinski, S. Mathur, H. Shen, T. Suszko, A. Gilewicz, B. Warcholinski // Applied Surface Science. - 2005. - Vol. 239, iss. 3-4. - P. 302-310. DOI: $10.1016 /$ j.apsusc.2004.05.278.

10. Materials properties of $\mathrm{ZnO} /$ diamond-like carbon (DLC) nanocomposite fabricated with different source of targets / Y. M. Foong, A. T. T. Koh, S. R. Lim, J. Hsieh, D. H. C. Chua // Diamond and Related Materials. - 2012. - Vol. 25. - P. 103-110. - DOI: 10.1016/j.diamond.2012.02.018.

11. Microstructure and tribological properties of magnetron sputtered nc-TiC/a-C nanocomposite / S. Zhang, X. L. Bui, J. Jiang, X. Li // Surface and Coatings Technology. - 2005. - Vol. 198, iss. 1-3. - P. 206-211. - DOI: 10.1016/j.surfcoat.2004.10.041.

12. Kelvin probe force microscopy and its application / W. Melitz, J. Shen, A. C. Kummel, S. Lee // Surface Science Reports. - 2011. - Vol. 66, iss. 1. - P. 1-27. DOI: $10.1016 /$ j.surfrep.2010.10.001.

13. Nonnenmacher M., O’Boyle M. P., Wickramasinghe H. K. Kelvin probe force microscopy // Applied Physics Letters. - 1991. - Vol. 58, iss. 25. - P. 2921-2923. - DOI: 10.1063/1.105227.

14. Nanocomposite vacuum-arc TiC/a-C:H coatings prepared using an additional ionization of acetylene / I. Sh. Trakhtenberg, N. V. Gavrilov, D. R. Emlin, S. A. Plotnikov, A. B. Vladimirov, E. G. Volkova, A. P. Rubshtein // The Physics of Metals and Metallography. - 2014. - Vol. 115, iss. 7. - P. 723-729. - DOI: 10.1134/S0031918X14070102.

15. The analysis of microhardness measurement approach for characterization of hard coatings / I. Sh. Trakhtenberg, A. B. Vladimirov, A. P. Rubstein, E. V. Kuzmina, K. Uemura, A. G. Gontar, S. N. Dub // Diamond and Related Materials. - 2003. - Vol. 12, iss. 10-11. - P. 1788-1792. DOI: $10.1016 / \mathrm{S} 0925-9635 Z ̌ 03.00288-7$.

Rubshtein A. P. et al. / Studying nanocomposite films with matrix-forming carbon by Kelvin probe force microscopy. 
16. Formation of morphology of surface of diamond-like films condenced upon arc deposition of graphite in a vacuum / I. Sh. Trakhtenberg, A. P. Rubshtein, A. B. Vladimirov, V. A. Yugov, S. A. Plotnikov, E. G. Volkova // The Physics of Metals and Metallography. - 2005. - Vol. 100, iss. 1. - P. 56-60.

17. Effect of hydrogen treatment on the field emission of amorphous carbon film / W. G. Xie, J. Chen, J. Chen, S. Z. Deng, J. C. She, N. S. Xu // Journal of Applied Physics. - 2007. - Vol. 101, iss. 8. - P. 084315-084500. - DOI: 10.1063/1.2724426.

18. Effect of work function and surface microstructure on field emission of tetrahedral amorphous carbon / A. Ilie, A. Hart, J. Flewitt, J. Robertson, W. I. Milne // Journal of Applied Physics. 2000. - Vol. 88, iss. 10. - P. 6002-6010. - DOI: 10.1063/1.1314874.

Rubshtein A. P. et al. / Studying nanocomposite films with matrix-forming carbon by Kelvin probe force microscopy. 